\title{
Study of Biodegradation of Film Materials with D-Cycloserine Based on Polyurethaneurea and the Dynamics of Drug Release
}

\author{
Tetiana Rudenchyk*, Rita Rozhnova, Nataliia Galatenko, Liudmyla Nechaeva \\ Department of Polymers of Medical Appointment, Institute of Macromolecular Chemistry of the National Academy of Sciences of Ukraine, \\ Kyiv, Ukraine
}

Email address:

rudenchyk@gmail.com (T. Rudenchyk),rozhnovarita@gmail.com (R. Rozhnova), politoks@merlin.net.ua (N. Galatenko)

${ }^{*}$ Corresponding author

\section{To cite this article:}

Tetiana Rudenchyk, Rita Rozhnova, Nataliia Galatenko, Liudmyla Nechaeva. Study of Biodegradation of Film Materials with D-Cycloserine Based on Polyurethaneurea and the Dynamics of Drug Release. American Journal of Polymer Science and Technology.

Vol. 5, No. 4, 2019, pp. 97-104. doi: 10.11648/j.ajpst.20190504.11

Received: October 1, 2019; Accepted: October 26, 2019; Published: November 8, 2019

\begin{abstract}
The study of the ability to biodegradation of film materials with D-cycloserine obtained on the basis of polyurethaneurea with fragments of the copolymer of N-vinylpyrrolidone with vinyl acetate and vinyl alcohol, and 1.6hexamethylenediamine in the structure under the influence of biological medium 199 for 1, 3 and 6 months were conducted. IR spectroscopy and physical-mechanical tests of these film materials before and after incubation in biological medium 199 were investigated. According to research results, there are decrease of the tensile strength (in 1.29-2.50 times) and a relative elongation at break (in 1.15-1.91 times) after incubation in biological medium 199. It is established that under the influence of biological medium 199 there are processes of biodegradation of film materials. The introduction of D-cycloserine into the composition of polyurethaneurea contributes to their biodegradation. It allows us to conclude that biodegradation is due to release of D-cycloserine. The study of the dynamics of D-cycloserine release from the polymer matrix was conducted by spectrophotometric method. The amount of drug varies depending on the copolymer content in structure of film materials (75.00-96.71\% of the total amount of the introduced drug). It has been established that the studied film materials are capable to the prolonged release of D-cycloserine. It allows using them as film coatings for medicine with different ability to release of drug depending on requirements.
\end{abstract}

Keywords: Polyurethaneurea, D-Cycloserine, N-vinylpyrrolidone Copolymer, Model Biological Medium 199, Biodegradation

\section{Introduction}

The polyurethaneurea (PUU) with fragments of the copolymer of N-vinylpyrrolidone with vinyl acetate and vinyl alcohol (VP-VA) thanks to biocompatibility, the increased hydrophilicity and ability of the prolonged release of drug from a polymer matrix it is expedient to use as polymeric carriers of drugs [1]. Therefore, we synthesized PUU based on diisocyanate prepolymer (DPP), 1.6hexamethylenediamine (HMDA) as a macrochain extender, and VP-VA copolymer as a hydrophilic component at different percentage ratio of diamine to copolymer and film materials with D-cycloserine on their basis [2].
The ability to biodegradation in the conditions imitating the environment of the organism is one of the most important characteristics of polymers for medical use as this process is accompanied by changes in the structure of the polymer which cause changes of properties and it can influence on the amount of released drug to the internal environment of an organism. It is known that the biodegradation processes are influenced by the chemical composition including a ratio of links of monomers, possible influence of drugs [3]. The ratio of components of polymer material, in particular, hydrophobic and hydrophilic component influences on the rate of biodegradation. An increase in the amount of hydrophilic component which absorbs more water leads to an 
increase in the rate of biodegradation $[4,5]$. The polymer matrixes containing water-soluble drugs demonstrate higher water absorption and higher ability to degradation whereas insoluble drugs slow down swelling and degradations of matrix [6].

Also important characteristic of polymers for medical use is the release dynamics of the drug and its quantity. The release of drugs from polymer composites is influenced by the chemical nature of the polymer and of drug, their physical-chemical properties, in particular the hydrophilicity of polymer matrix [7]. It is known what for hydrophilic polymer matrixes the release of drugs increases and is controllable in comparison with hydrophobic [8,9]. Since hydrophilic polymers have high ability to hydration and swelling, it contributes to the formation of a gel layer, which is a surface barrier and prevents the movement of the drug from the inside layer of the polymer and provides its controlled release without burst release $[10,7]$. It is also known that an increase in the content of a hydrophilic component leads to an increase in the rate of release and the amount of released drug [5]. Hydrophilic polymer matrices are systems in which two competing release mechanisms are involved. First of all the kinetics of drug release depends on diffusion. However, degradation of polymer also promotes the release of drug [11]. The authors report that increasing the rate of degradation leads to an increase in the rate of release of the drug and its amount [5]. Such factor as water solubility of drug determines the mechanism and kinetics of its release from a polymer matrix. Well water soluble drugs are released due to diffusion that promotes swelling of a hydrophilic matrix and degradation of polymer, while poorly water-soluble drugs are released mainly due to degradation of polymer matrix $[12,13]$.

All these parameters influencing degradation and release of drugs should be taken into account when creating prolonged-release preparations to ensure a uniform release of drugs to an organism in an acceptable quantity, which will be sufficient for the manifestation of biological activity and will not exert a toxic effect.

Considering the aforesaid, there is a need to study in vitro biodegradation and release dynamics of drug from the polymer matrix of studied film materials. Results of these researches will allow predicting behavior of polymer materials at their further application in medical practice.

Therefore, the purpose of the work is to study the biodegradation of film materials with D-cycloserine based on PUU with hydrophilic VP-VA copolymer and HMDA fragments synthesized at different ratio of components and the release dynamics of drug from the polymer matrix.

\section{Experimental}

\subsection{Materials}

The PUU with hydrophilic VP-VA copolymer and HMDA fragments in the structure synthesized at different percentages ratio of HMDA: VP-VA (40:60, 50:50, 60:40,
70:30) and film materials filled by D-cycloserine (in the amount of 1 wt. \%) on their basis were objects of researches [2].

Biological medium 199 (BM 199) (pH 7.4-7.7), which is a complex mixture of proteins, amino acids, carbohydrates, fats, salts, hormones, enzymes and soluble gases was selected as a model medium to study biodegradation. BM 199 was purchased from BioTestLab (Ukraine).

Distilled water and drug D-cycloserine (molecular weight $=102.09$, melting temperature $=147^{\circ} \mathrm{C}$ ) were used to study the dynamics of $\mathrm{D}$-cycloserine release. D-cycloserine was purchased from Merck.

\subsection{The Method of Incubation in BM 199}

Samples of PUU in the form of $0.5 \times 5.0 \mathrm{~cm}$ strips were placed in sterile tubes, poured $25 \mathrm{ml}$ of model biological medium and kept in a thermostat at a temperature of $(37 \pm$ $1)^{\circ} \mathrm{C}$ for 1,3 and 6 months. Solutions of model mediums were changed daily. After defined incubation terms in the model medium, the samples were taken out, washed with distilled water and dried to a constant masse at $T=70^{\circ} \mathrm{C}$.

\subsection{Study Methods}

\subsubsection{Fourier Transforms Infrared (ATR FT-IR) Spectroscopy}

The structure of samples was investigated on a Tensor-37 FT-IR spectrometer in the range $650-4000 \mathrm{~cm}^{-1}$ by the MATR method with the aid of a diamond crystal trapezoidal prism (a number of reflections of $\mathrm{N}=1$, an incidence angle of $\left.\varphi=39^{\circ}\right)$. The absorption bands were assigned as described in [14].

\subsubsection{Mechanical Tests}

The physical-mechanical properties such as tensile strength at break $(\sigma, \mathrm{MPa})$ and relative elongation at break $(\varepsilon, \%)$ were determined on the tension testing machine P 5 in accordance with GOST 25.601.

The tensile strength at break $(\sigma)$ was calculated using Equation (1):

$$
\sigma=\frac{F_{\max }}{b \cdot h}
$$

Where: $F_{\max }-$ maximum load at break of sample, $\mathrm{H}$;

$b$ - width of sample, $\mathrm{mm}$;

$\mathrm{h}$ - thickness of sample, $\mathrm{mm}$.

The relative elongation at break $(E)$ was calculated using Equation (2):

$$
E=\frac{\Delta l}{l} \cdot 100
$$

Where: $\Delta l$ - absolute elongation of sample at break, $\mathrm{mm}$; $l$ - initial length of sample, $\mathrm{mm}$.

\subsubsection{Dynamics of D-Cycloserine Release Study}

The study of the D-cycloserine release from polymer samples was performed by spectrophotometric method. 
Absorption spectra of the studied solutions were obtained on the "SPECORD M-40" device in cuvettes with a layer thickness of $1 \mathrm{~cm}$ relative to the comparison solutions.

Preparation of solutions. Samples (average weight $0.3 \mathrm{~g}$ ) were placed in weighing bottles with the ground-in stopper, added $20 \mathrm{ml}$ of distilled water. The weighing bottles were kept in a thermostat at a temperature $(38 \pm 1){ }^{\circ} \mathrm{C}$ for $(1,7,14$, 21 and 28) days. After defined terms the solutions were poured out and investigated their absorption spectra. The studied solutions received from the samples containing Dcycloserine in their composition. Solutions of comparative (control solutions) obtained from control samples (from samples which do not contain D-cycloserine).

The concentration of released D-cycloserine was found on the calibration graph. For creation of the calibration graph of dependence of optical density of solutions on D-cycloserine concentration, a series of D-cycloserine solutions with different percentage concentration: $0.0005 ; 0.00075$; 0.0010 ; $0.0015 ; 0.0020$ were prepared. Their absorption spectra were investigated.

The amount of D-cycloserine released into the solution from polymer samples $(C S, \%)$ was calculated using Equation (3) and (4):

$$
\begin{aligned}
& C S(\%)=\frac{m}{m_{o}} \cdot 100 \%, \\
& m=\frac{C \cdot M \cdot V}{1000}
\end{aligned}
$$

Where: $C S(\%)$ - amount of D-cycloserine released from the polymer samples into the solution, $\%$;

$m$ - amount of D-cycloserine released from the polymer for certain period of time, $\mathrm{g}$;

$m_{0}$ - masse of D-cycloserine introduced into the polymer, g;

$C$ - concentration of D-cycloserine in the investigated solution, found according to the calibration graph, $\mathrm{mol} / \mathrm{L}$;

$M$ - molecular weight of D-cycloserine;

$V$ - volume of the solution in which performed washingout, $\mathrm{ml}$.

\section{Results and Discussion}

The ability to biodegradation of PUU with hydrophilic VPVA copolymer fragments and a macrochain extender HMDA in the structure, and film materials with D-cycloserine obtained on their basis after their incubation in model biological medium imitating the environment of the organism human was investigated by IR spectroscopy method and by changing their physical-mechanical parameters before (control) and after incubation in BM 199 for 1,3 and 6 months.

\subsection{Study of Biodegradation by Physical-mechanical Tests}

Physical-mechanical tests, namely changes in tensile strength $(\sigma, \mathrm{MPa})$ and relative elongation at break $(\varepsilon, \%)$ of polymer materials based on DPP, HMDA, VP-VA and Dcycloserine (in the amount of 1 wt. \%) obtained at different percentage ratio of HMDA: VP-VA (40:60; 50:50; 60:40; $70: 30$ ) after an incubation in model biological medium for 1 , 3 and 6 months have been conducted.

According to the results of studies of physical-mechanical properties after incubation in BM 199 of PUU with VP-VA copolymer fragments without the contents of a drug there is a slight increase of tensile strength at break after 1 month of incubation for all samples (Table 1).

The tensile strength at break of PUU with fragments of VP-VA copolymer before the incubation in BM 199 are in the range of 1.6-2.8 $\mathrm{MPa}$, whereas after 6 months of incubation are in the range of 1.5-4.1 $\mathrm{MPa}$ (Table 1). Thus, after 6 months of incubation in BM 199 there is an increase of tensile strength at break of PUU with VP-VA copolymer fragments compared with the control. The exception is PUU with maximum content of VP-VA copolymer in the structure (synthesized by the percentage ratio of HMDA: VP-VA = 40:60), which lose strength characteristics in 1.27 times after 6 months of incubation. Thus, a tendency has been revealed namely, when increasing the copolymer content in the structure of PUU there is an increase in the tensile strength at break to a lesser extent, and when the maximum amount of copolymer $(60 \%)$ there is its decrease.

\begin{tabular}{|c|c|c|c|c|c|}
\hline \multirow{2}{*}{ Samples } & \multirow{2}{*}{ HMDA/VP-VA, \% } & \multicolumn{4}{|c|}{ Tensile strength at break ( $\sigma), \mathrm{MPa}$} \\
\hline & & control & 1 month & 3 months & 6 months \\
\hline \multirow{4}{*}{$\mathrm{DPP}+\mathrm{HMDA}+\mathrm{VP}-\mathrm{VA}$} & $40: 60$ & 1.9 & 2.1 & 2.1 & 1.5 \\
\hline & $50: 50$ & 1.6 & 2.3 & 2.3 & 2.1 \\
\hline & $60: 40$ & 2.0 & 2.5 & 2.8 & 2.7 \\
\hline & $70: 30$ & 2.8 & 3.7 & 4.0 & 4.1 \\
\hline \multirow{4}{*}{$\mathrm{DPP}+\mathrm{HMDA}+\mathrm{VP}-\mathrm{VA}+\mathrm{D}-\mathrm{cycloserine}$} & $40: 60$ & 2.7 & 2.6 & 2.2 & 1.6 \\
\hline & $50: 50$ & 2.0 & 2.3 & 1.5 & 0.8 \\
\hline & $60: 40$ & 2.9 & 2.7 & 3.2 & 1.5 \\
\hline & $70: 30$ & 3.1 & 3.2 & 3.9 & 2.4 \\
\hline
\end{tabular}

Table 1. Tensile strength at break of PUU and film materials with D-cycloserine after incubation in BM 199.

The tensile strength at break of film materials with Dcycloserine, which obtained based on PUU with VP-VA copolymer fragments in the structure before the incubation in BM 199 is 2.0-3.1 MPa, whereas after 6 months of incubation is $0.8-2.4 \mathrm{MPa}$ (Table 1). So under the influence of biological medium 199 it is the decrease of the tensile strength at break of all film materials with D-cycloserine in 1.29-2.50 times compared with the control.

Relative elongation at break of PUU with VP-VA copolymer fragments before the incubation in BM 199 are in 
the range of $27-46 \%$, whereas after 6 months of incubation are in the range of $22-81 \%$ (Table 2). For PUU with the minimum content of the VP-VA copolymer in the structure (synthesized by the percentage ratio of HMDA: VP-VA = 70:30) after 6 months of incubation in BM 199 there is an increase in relative elongation at break. With an increase in the content of copolymer in the structure of the PUU after 6 months of incubation there is a decrease of the relative elongation at break in 1.23-1.73 times.

Table 2. Relative elongation at break of PUU and film materials with D-cycloserine after incubation in BM 199.

\begin{tabular}{|c|c|c|c|c|c|}
\hline \multirow{2}{*}{ Samples } & \multirow{2}{*}{ HMDA/VP-VA, \% } & \multicolumn{4}{|c|}{ Relative elongation at break $(\varepsilon), \%$} \\
\hline & & control & 1 month & 3 months & 6 months \\
\hline \multirow{4}{*}{ DPP+HMDA+VP-VA } & $40: 60$ & 38 & 43 & 29 & 22 \\
\hline & $50: 50$ & 27 & 39 & 34 & 22 \\
\hline & $60: 40$ & 37 & 42 & 32 & 29 \\
\hline & $70: 30$ & 46 & 80 & 73 & 81 \\
\hline \multirow{3}{*}{$\mathrm{DPP}+\mathrm{HMDA}+\mathrm{VP}-\mathrm{VA}+\mathrm{D}$-cycloserine } & $40: 60$ & 63 & 47 & 32 & 33 \\
\hline & $50: 50$ & 37 & 33 & 30 & 25 \\
\hline & $70: 30$ & 47 & 53 & 61 & 36 \\
\hline
\end{tabular}

Relative elongation at break of film materials with Dcycloserine based on PUU with VP-VA copolymer fragments in the structure before the incubation in BM 199 is 37-63\%, whereas after 6 months of incubation is $25-39 \%$ (Table 2). That is for all samples there is a decrease in the relative elongation at break 1.15-1.91 times.

Thus, above-mentioned changes after the incubation in BM 199 of PUU and film materials with drug on their basis allow us to conclude about the influence of the concentration of VP-VA copolymer in the structure of the polymer matrix on their physical-mechanical properties. So, at minimum content of copolymer in the structure of the PUU under the influence of BM 199 there is an increase of strength characteristics, while increasing the copolymer content causes a decrease of strength (at $60 \%$ of VP-VA) and relative elongation at break (from $40 \%$ of VP-VA). It is confirmed by the well-known data that increase in amount of a hydrophilic component leads to increase of biodegradation $[4,5]$. For all film materials with D-cycloserine based on PUU with fragments of copolymer VP-VA after the incubation in BM 199 there is a decrease of tensile strength and relative elongation at break. It allows concluding that the introduction of D-cycloserine into the PUU contributes to their biodegradation, which is probably due to release of Dcycloserine from the polymer matrix. This is because Dcycloserine is water-soluble drug. Therefore its release happens due to diffusion which promotes penetration of BM 199 into a polymer matrix and its swelling. And it in turn promotes to degradation of polymer material $[12,13]$.

\subsection{Study of Biodegradation by ATR FT-IR Spectroscopy}

In order to determine the influence of BM 199 on the structure of investigated polymer materials, IR-spectroscopic investigations of samples before the incubation (control) and samples after incubation in BM 199 were conducted. To detect the influence of D-cycloserine on the process of biodegradation of film materials, infrared spectra of PUU with hydrophilic fragments of VP-VA copolymer in the structure and film materials with D-cycloserine on their basis were removed. To detect the influence of the chemical composition on the process of biodegradation of polymer materials, the infrared spectra of PUU synthesized at different percentages ratio of component were removed.

Infrared spectra of PUU with fragments of VP-VA copolymer in structure synthesized by the percentage ratio HMDA: VP-VA 70:30 after incubation in BM 199 remained unchanged (Figure 1).

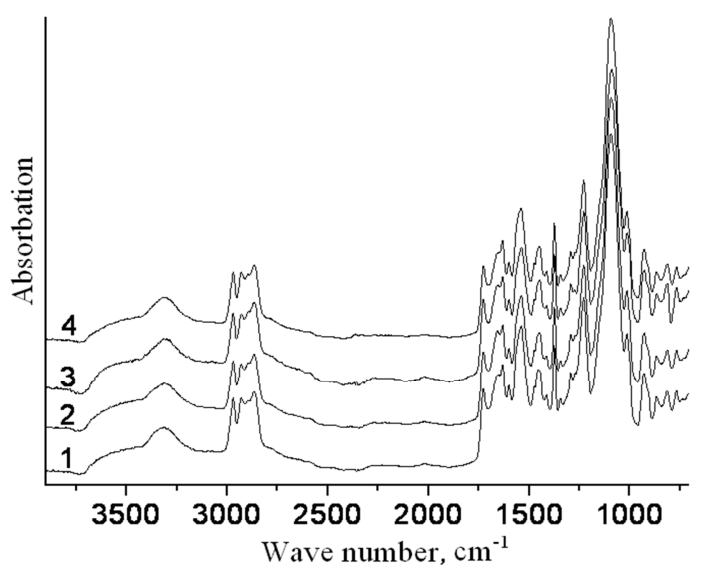

Figure 1. IR-spectra of DPP+HMDA+VP-VA (70:30) before (1) and after incubation in BM 199 for 1 month (2), 3 months (3), 6 months (4).

On the infrared spectra of film materials with D-cycloserine which are received based on PUU synthesized at the percentage ratio of HMDA: VP-VA = 70:30 after incubation in BM 199 there is a decrease of intensity of the absorption band $v_{\mathrm{NH}-\text { free }}$ with approximate maximum $3515 \mathrm{~cm}^{-1}$ and narrowing of the absorption band $v_{\mathrm{NH}-b o n d}$ with a maximum $3316 \mathrm{~cm}^{-1}$ with a simultaneous increase of its intensity (Figure 2). It demonstrates an increase in the number of NH groups bound by hydrogen bonds. The intensity of the absorption band $v_{\mathrm{C}=\mathrm{O}}$ of vinylpyrrolidone ring with a maximum $1660 \mathrm{~cm}^{-1}$ during incubation in model biological medium gradually decreases, and the intensity of the absorption band deformation vibrations of $\mathrm{NH}$ groups with a maximum $1632 \mathrm{~cm}^{-1}$ increases (Figure 2). It is connected with increase in the number of $\mathrm{NH}$ groups on the surface of the samples due to spatial changes in the surface layer molecules (as IR spectra are removed from the surface of polymer materials). 


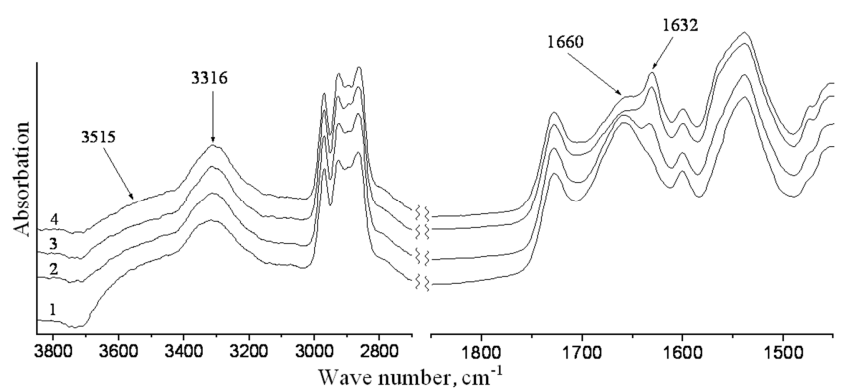

Figure 2. IR-spectra of film materials with D-cycloserine obtained based on $D P P+H M D A+V P-V A$ (70:30) before (1) and after incubation in BM 199 for 1 month (2), 3 months (3), 6 months (4).

After incubation in BM 199 on the infra-red spectra of PUU synthesized at the percentage ratio of HMDA: VP-VA = 50:50 (Figure 3) and on the infra-red spectra of film materials with D-cycloserine obtained on their basis (Figure 4) the changes are identical. The infra-red spectra demonstrate the decrease of the intensity of the absorption band $v_{\mathrm{NH} \text {-free }}(3515$ $\left.\mathrm{cm}^{-1}\right)$, the narrowing of the absorption band $v_{\mathrm{NH}-b o n d}(3316$ $\mathrm{cm}^{-1}$ ) with a simultaneous increase its intensity, the decrease of the intensity of the absorption band $v_{\mathrm{C}=\mathrm{O}}$ vinylpyrrolidone ring $\left(1660 \mathrm{~cm}^{-1}\right)$ and the increase of the intensity of the absorption band deformation vibrations of $\mathrm{NH}$ groups (1632 $\mathrm{cm}^{-1}$ ) (Figure 3, Figure 4).

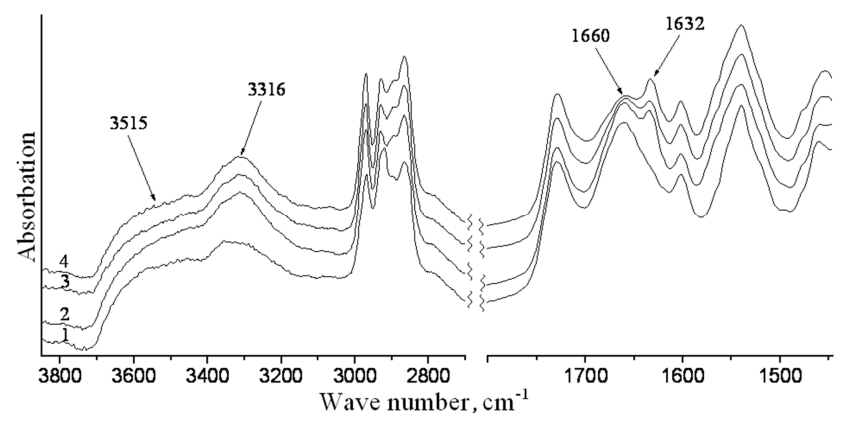

Figure 3. IR-spectra of DPP+HMDA+VP-VA (50:50) before (1) and after incubation in BM 199 for 1 month (2), 3 months (3), 6 months (4).

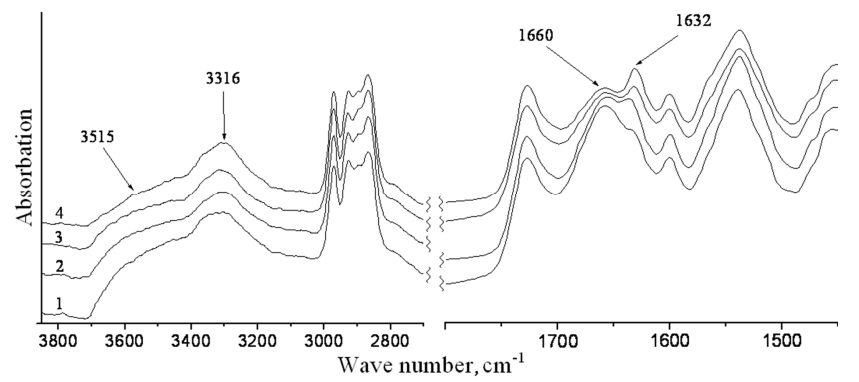

Figure 4. IR-spectra of film materials with D-cycloserine obtained based on $D P P+H M D A+V P-V A$ (50:50) before (1) and after incubation in BM 199 for 1 month (2), 3 months (3), 6 months (4).

Thus, according to the results of IR spectroscopy, we can conclude that after incubation of the studied materials in BM 199 there is a decrease of intensity of the absorption band $v_{\mathrm{NH}-\text { free }}$ and narrowing of the absorption band $v_{\mathrm{NH}-b o n d}$ with a simultaneous increase of its intensity (for PUU (50:50) and all film materials with D-cycloserine). It demonstrates an increase in the number of $\mathrm{NH}$ groups bound by hydrogen bonds. There is also a decrease of the intensity of the absorption band $v_{\mathrm{C}=\mathrm{O}}$ of vinylpyrrolidone ring, which is likely to indicate the processes of biodegradation and increase of the intensity of the absorption band deformation vibrations of $\mathrm{NH}$ groups (for PUU (50:50) and all film materials with Dcycloserine), which is connected with increase in the number of NH groups on the surface of the samples due to spatial changes in the surface layer molecules. Thus, under the influence of BM 199 there are processes of biodegradation, which are accompanied by the redistribution of hydrogen bonds between NH groups of polymer matrix. The results of the studies allowed determining the dependence of the abovementioned changes from the presence of D-cycloserine in the composition of PUU and from the concentration of VP-VA copolymer in their structure. The changes on the infrared spectra which indicate about biodegradation are observed for samples of PUU with high content of copolymer in the structure $(50 \%)$ and for all film materials with D-cycloserine. Thus, the processes of biodegradation of PUU and film materials with D-cycloserine are influenced by the amount of the hydrophilic component in their structure and the presence of the drug that is consistent with the results of physicalmechanical researches.

To date the biodegradability of polymer materials containing $\mathrm{N}$-vinylpyrrolidone, vinyl acetate, vinyl alcohol in the structure has been sufficiently investigated [15-17]. However, studies of biodegradation of polymer materials containing of the ternary copolymer of $\mathrm{N}$-vinylpyrrolidone with vinyl acetate and vinyl alcohol in the structure are scarce.

The results of our studies are consistent with the results of biodegradability studies of film materials with antibiotic tiamulin fumarate based on hydrophilic PUU synthesized at different ratio of VP-VA and macrochain extender HMDA in the structure [18]. According to the results of the tests, it is established that after incubation in BM 199 of film materials with tiamulin fumarate there are processes of biodegradation of the polymer matrix. It has also been found that increasing the amount of hydrophilic component in the structure of the film materials and the presence of the drug substance contributes to their biodegradation.

\subsection{Investigation of Dynamics of D-Cycloserine Release}

Obtained results of physical-mechanical tests allowed concluding that the release of D-cycloserine from the polymer matrix is probable. However, quantitative proofs are necessary for polymers of medical appointment. Therefore, the study of its release was carried out by spectrophotometric method.

The film materials with D-cycloserine (in the amount of 1 wt. \%) obtained based on PUU with hydrophilic VP-VA copolymer and a macrochain extender HMDA in the structure synthesized at different percentages ratio of HMDA: VP-VA were objects of researches of the dynamics of Dcycloserine release.

For statistical reliability we investigated 3 samples of each 
film material with D-cycloserine and a control sample containing all components in the same amounts excepting a cycloserine.

The value of the optical density of D-cycloserine solutions at the maximum of the band at wavelength $\lambda=(224 \pm 1) \mathrm{nm}$ are presented in Table. 3 .

Table 3. Spectrophotometric study of D-cycloserine solutions for creation of the calibration graph.

\begin{tabular}{lll}
\hline $\begin{array}{l}\text { Solution concentrations } \\
\text { of D-cycloserine }(\mathbf{C}), \boldsymbol{\%}\end{array}$ & Wavelength $(\boldsymbol{\lambda}), \mathbf{n m}$ & Optical density $\left(\mathbf{D}_{\text {avg. }}\right)$ \\
\hline 0.00050 & 223.2 & 0.200 \\
0.00075 & 224.4 & 0.296 \\
0.00100 & 223.4 & 0.391 \\
0.00150 & 224.6 & 0.546 \\
0.00200 & 224.2 & 0.738 \\
\hline
\end{tabular}

The calibration graph of dependence of the optical density on concentration of D-cycloserine solutions is a straight line passing through the origin of coordinates (Figure 5).

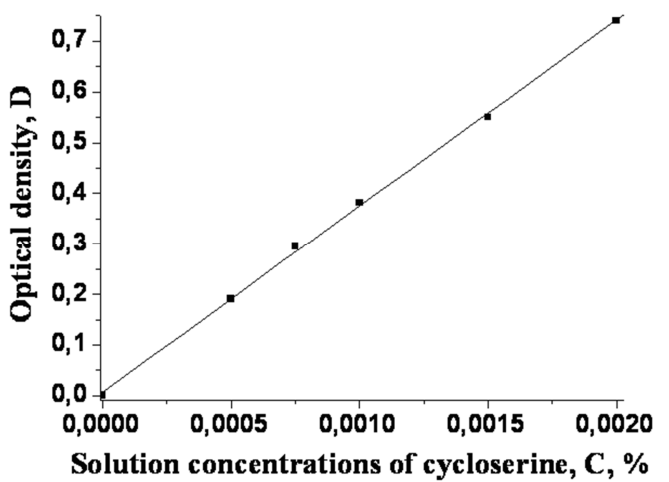

Figure 5. Graph of dependence of the optical density on concentration of Dcycloserine solutions.

The results of measurements and calculations of D-cycloserine release from polymer samples are presented in Table. 4.

Table 4. The results of the study of the dynamics of the D-cycloserine release.

\begin{tabular}{|c|c|c|c|c|c|}
\hline \multirow{2}{*}{$\begin{array}{l}\text { Washing-out } \\
\text { time, day }\end{array}$} & \multirow{2}{*}{$\begin{array}{l}\text { Optical density of } \\
\text { solution ( }\left(_{\text {avg. }}\right)\end{array}$} & \multirow{2}{*}{$\begin{array}{l}\text { Concentration of } D \text { - } \\
\text { cycloserine (according to the } \\
\text { calibration graph) (C), \% }\end{array}$} & \multicolumn{3}{|c|}{ The amount of D-cycloserine released into the solution from polymer sample } \\
\hline & & & $\begin{array}{l}\text { from the moment of the } \\
\text { previous definition }(\mathrm{m}), \mathrm{g}\end{array}$ & $\begin{array}{l}\text { from the beginning of } \\
\text { washing-out }(\mathrm{m}), \mathrm{g}\end{array}$ & $\begin{array}{l}\% \text { from introduced } \\
\text { D-cycloserine, } \%\end{array}$ \\
\hline \multicolumn{6}{|c|}{ DPP+HMDA+VP-VA $(70: 30)$} \\
\hline 1 & 0.6548 & 0.0017 & 0.00102 & 0.00102 & 33.55 \\
\hline 7 & 0.5755 & 0.0015 & 0.00060 & 0.00162 & 53.29 \\
\hline 14 & 0.8850 & 0.0023 & 0.00046 & 0.00208 & 68.42 \\
\hline 21 & 0.2792 & 0.0007 & 0.00014 & 0.00222 & 73.03 \\
\hline \multicolumn{6}{|c|}{ DPP+HMDA+VP-VA $(60: 40)$} \\
\hline 1 & 0.3349 & 0.0009 & 0.00108 & 0.00108 & 34.18 \\
\hline 7 & 0.7249 & 0.0019 & 0.00076 & 0.00184 & 58.23 \\
\hline 14 & 0.6423 & 0.0017 & 0.00067 & 0.00252 & 79.74 \\
\hline 21 & 0.3401 & 0.0009 & 0.00018 & 0.00270 & 85.00 \\
\hline 28 & 0.1198 & 0.0003 & 0.00006 & 0.00276 & 87.34 \\
\hline \multicolumn{6}{|c|}{$\mathrm{DPP}+\mathrm{HMDA}+\mathrm{VP}-\mathrm{VA}(50: 50)$} \\
\hline 14 & 0.6175 & 0.0016 & 0.00064 & 0.00256 & 82.31 \\
\hline 21 & 0.2328 & 0.0006 & 0.00012 & 0.00268 & 86.17 \\
\hline 28 & 0.1605 & 0.0004 & 0.00008 & 0.00276 & 88.74 \\
\hline \multicolumn{6}{|c|}{$\mathrm{DPP}+\mathrm{HMDA}+\mathrm{VP}-\mathrm{VA}(40: 60)$} \\
\hline 1 & 0.4361 & 0.0011 & 0.00132 & 0.00132 & 42.03 \\
\hline 7 & 0.5014 & 0.0013 & 0.00104 & 0.00236 & 75.16 \\
\hline 14 & 0.3496 & 0.0009 & 0.00036 & 0.00272 & 86.62 \\
\hline 21 & 0.1522 & 0.0004 & 0.00008 & 0.00280 & 89.17 \\
\hline 28 & 0.1916 & 0.0005 & 0.00010 & 0.00290 & 92.36 \\
\hline \multicolumn{6}{|c|}{ DPP+HMDA+VP-VA $(30: 70)$} \\
\hline 1 & 0.5081 & 0.0013 & 0.00156 & 0.00156 & 51.32 \\
\hline 7 & 0.4582 & 0.0012 & 0.00096 & 0.00252 & 82.89 \\
\hline 14 & 0.2836 & 0.0007 & 0.00028 & 0.00280 & 97.10 \\
\hline 21 & 0.1141 & 0.0003 & 0.00006 & 0.00286 & 94.07 \\
\hline 28 & 0.1513 & 0.0004 & 0.00008 & 0.00294 & 96.71 \\
\hline
\end{tabular}

According to Table 4, the ratio of HMDA: VP-VA in the structure of the polymer influences on the dynamics of Dcycloserine release from the polymer matrix. It has been established that when the content of VP-VA copolymer in the structure of PUU increasing, the amount of released Dcycloserine increases. These results are coordinated with the known data that the increase of hydrophilicity leads to increase in the amount of released drug [5]. It is known that the studied PUU are hydrophilic, and increase of content of hydrophilic VP-VA copolymer in their structure leads to increase water absorption [2].

The D-cycloserine release from the polymer matrix of film materials synthesized for different contents of the copolymer is in the range from 75.00 to $96.71 \%$ of the total amount of introduced drug for 28 days. Incubation of samples of film materials with a maximum content of VP-VA copolymer 
$($ DPP + HMDA + VP-VA (30:70)) during 28 days leads to the destruction of the polymer basis and the release of almost $97 \%$ of the introduced cycloserine. It can be explained by the biodegradation of polymer materials. After all by results of our studies an increase of copolymer contents there is an increase of biodegradation. And the increase of degradation rate of polymer matrix leads to increase of release rate of drug and its quantity [5].

Graphically the results of the dynamics of release are presented in Figure 6.

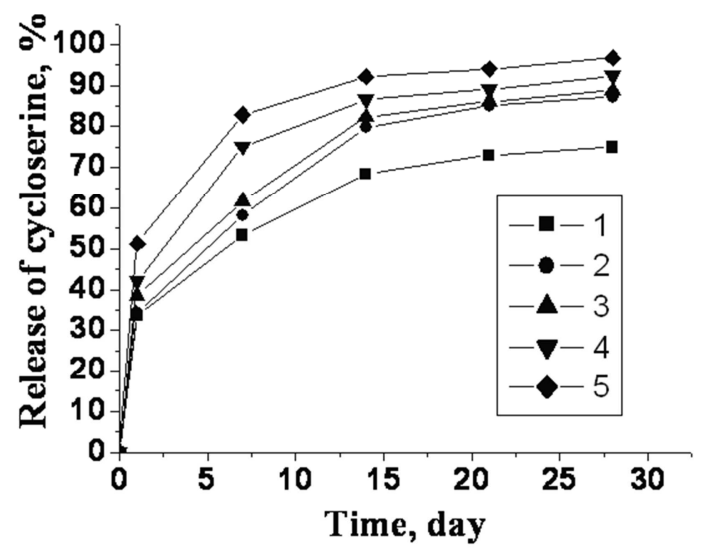

Figure 6. The dynamics of D-cycloserine release from samples of film materials obtained at different ratio of HMDA: VP-VA: 70:30 (1), 60:40 (2), 50:50 (3), 40:60 (4), 30:70 (5).

So, according to the results of researches of dynamics of D-cycloserine release, the film materials based on PUU with fragments of VP-VA copolymer and HMDA in the structure are capable of prolonged release of D-cycloserine. On the basis of the obtained results we can conclude that the release of D-cycloserine occurs due to two mechanisms: diffusion of water soluble drug and biodegradation of polymer matrix. The release of D-cycloserine from the polymer matrix is influenced by the concentration of hydrophilic VP-VA copolymer in the structure of the PUU. Thus, film coatings for medicine with different ability to release of drug depending on the need can be obtained by variation of content of the VP-VA copolymer in the structure of PUU.

The obtained results (Table 4) demonstrate that 0,00102$0,00156 \mathrm{~g}$ of D-cycloserine in $20 \mathrm{ml}$ of solution which is 51 $78 \mu \mathrm{g} / \mathrm{ml}$ is released for film materials with different contents of the VP-VA copolymer during the first day. During the next 6 days $0.00060-0.00104 \mathrm{~g}$ in $20 \mathrm{ml}(30-52 \mu \mathrm{g} / \mathrm{ml})$ is released, and from 7 th to 14 th day $0.00028-0.00067 \mathrm{~g}$ in $20 \mathrm{ml}(14-$ $33.5 \mu \mathrm{g} / \mathrm{ml})$ is released. For the next 7 days $0.00006-0.00018$ $\mathrm{g}$ in $20 \mathrm{ml}(3-9 \mu \mathrm{g} / \mathrm{ml})$ is released. For the last term from the $21 \mathrm{st}$ to the 28 th day $0.00006-0.00010 \mathrm{~g}$ in $20 \mathrm{ml}(3-5 \mu \mathrm{g} / \mathrm{ml})$ is released.

It is known that the minimum concentration of Dcycloserine which suppresses Mycobacterium tuberculosis is $5-25 \mu \mathrm{g} / \mathrm{ml}[19,20], 1.5-30 \mu \mathrm{g} / \mathrm{ml}$ depending on the medium of culture used [21]. That is the amount of D-cycloserine released from the studied film materials is sufficient for the manifestation of bacteriostatic activity. Besides, the maximum concentration of the released drug is not superfluous and toxic. It is proved by the results of tissue culture studies and histological tests which demonstrate absence of toxic action on the cultured cells and a biocompatibility with tissues of experimental animals [22].

It is also known that D-cycloserine is a broad-spectrum antibiotic. It has a bacteriostatic and bactericidal action depending on the concentration of the drug in the place of inflammation and the sensitivity of the microorganisms [23, 24]. It is active in relation to a number of gram-positive and gram-negative microorganisms. The antibiotic inhibits the growth in vitro of Escherichia coli, Staphylococcus aureus, Enterococcus, Nocardia and Chlamydia species [25, 26], Enterobacteriaceae [20] and other species of microorganisms. The minimum inhibitory concentration of D-cycloserine is 16-64 $\mu \mathrm{g} / \mathrm{ml}$ for Escherichia coli, $64-128 \mu \mathrm{g} / \mathrm{ml}$ for grampositive bacteria Enterococcus faecium [27], 8-64 $\mu \mathrm{g} / \mathrm{ml}$ for Staphylococcus aureus $[27,28]$. The minimum concentration of antibiotic that inhibits the growth of bacteria in relation to gram-negative Enterobacteriaceae is $2-100 \mu \mathrm{g} / \mathrm{ml}$ and more [24]. However, to increase the inhibiting action on Pseudomonas spp, higher concentrations of D-cycloserine are necessary $(400 \mu \mathrm{g} / \mathrm{ml})$ [23].

Considering the aforesaid, D-cycloserine is released from the studied film materials in an amount that will be sufficient for the manifestation of bacteriostatic and bactericidal activity.

\section{Conclusions}

The ability to biodegradation of film materials with Dcycloserine based on polyurethaneurea with fragments of copolymer of $\mathrm{N}$-vinylpyrrolidone with vinyl acetate and vinyl alcohol, and 1.6-hexamethylenediamine in the structure under the influence of biological medium 199 for 1,3 and 6 months was investigated. It is established that under the influence of biological medium 199 there are processes of biodegradation of film materials, which are confirmed by a decrease in the intensity of the absorption band $v_{\mathrm{C}=\mathrm{O}}$ of vinylpyrrolidone ring and by a decrease of the tensile strength and a relative elongation at break. Along with biodegradation there is a redistribution of hydrogen bonds between NH-groups of the polymer matrix. Decrease the physical-mechanical properties of film materials are due to the D-cycloserine release and it indicates that the introduction of D-cycloserine into the composition of polyurethaneurea contributes to their biodegradation. By results of the dynamics of D-cycloserine release from the polymer matrix it has been established that the studied film materials are capable to the prolonged release of the drug, the amount of which varies depending on the copolymer content in their structure $(75.00-96.71 \%$ of the total amount of introduced drug). Considering the aforesaid, the studied film materials with D-cycloserine can be proposed for use as film coatings for the treatment of skin tuberculosis. 


\section{References}

[1] Rudenchyk T., Rozhnova R., Galatenko N. Hydrophylic polyurethaneurea containing the copolymer of $\mathrm{N}$ vinylpyrrolidone, vinyl acetate and vinyl alcohol for possible biomedical use. Advances in Biochemistry, 5, 7378 (2017).

[2] Rudenchyk T. V., Rozhnova R. A., Galatenko N. A., Kiselova T. O. Hydrophilic polyurethane ureas with cycloserine which contain in their structure the fragments of a copolymer of $\mathrm{N}$ vinylpyrrolidone with vinyl alcohol: synthesis and characterization. Voprosy Khimii i Khimicheskoi Tekhnologii, No. 5, 49-57 (2017).

[3] Makadia H. K., Siegel S. J. Poly lactic-co-glycolic acid (PLGA) as biodegradable controlled drug delivery carrier. Polymers (Basel), 3, 1377-1397 (2011).

[4] Ansary R. H., Awang M. B., Rahman M. M. Biodegradable poly (D, L-lactic-co-glycolic acid) -based micro/nanoparticles for sustained release of protein drugs - A review. Tropical Journal of Pharmaceutical Research, 13, 1179-1190 (2014).

[5] Alexis F. Factors affecting the degradation and drug-release mechanism of poly (lactic acid) and poly [(lactic acid)-co(glycolic acid)]. Polymer International, 54, 36-46 (2005).

[6] Chakraborty S., Khandai M., Sharma A., Patra Ch., Patro V., Sen K. Effects of drug solubility on the release kinetics of water soluble and insoluble drugs from HPMC based matrix formulations. Acta Pharmaceutica, 59, 313-323 (2009).

[7] Reza M. S., Quadir M. A., Haider S. S. Comparative evaluation of plastic, hydrophobic and hydrophilic polymers as matrices for controlled-release drug delivery. Journal of Pharmacy and Pharmaceutical Sciences, 6, 282-291 (2003).

[8] Anepu S., Duppala L., Pratyusha P. V. Development of hydrophobic carriers based tablets for sustained release of verapamil. Journal of Applied Pharmaceutical Science, 5, 125134 (2015).

[9] Brazel C. S., Peppas N. A. Mechanisms of solute and drug transport in relaxing, swellable, hydrophilic glassy polymers. Polymer, 40, 3383-3398 (1999).

[10] Ali T., Shoaib M. H., Yousuf R. I., Jabeen S., Muhammad I. N., Tariq A. Use of hydrophilic and hydrophobic polymers for the development of controlled release tizanidine matrix tablets Brazilian Journal of Pharmaceutical Sciences, 50, 779-818 (2014).

[11] Nisha S., Mathew G., Lincy J. Matrix tablets: an effective way for oral controlled release drug delivery. Iranian Journal of Pharmaceutical Sciences, 8, 165-170 (2012).

[12] Varma M. V., Kaushal A. M., Garg A., Garg S. Factors affecting mechanism and kinetics of drug release from matrixbased oral controlled drug delivery systems. American Journal of Drug Delivery, 2, 43-57 (2004).

[13] Tiwari S. B., Rajabi-Siahboomi A. R. Extended-release oral drug delivery technologies: monolithic matrix systems. In: 'Drug Delivery Systems' Vol. 437. Methods in molecular biology (Ed.: Jain K. K.) Humana Press, Totowa, NJ 217-243 (2008).

[14] Pretsch E., Bëllmann P., Affolter C. Structure determination of organic compounds. Tables of spectral data. Springer-Verlag Berlin Heidelberg New York (2000).

[15] Amann M., Minge O. Biodegradability of Poly (vinyl acetate) and Related Polymers. Advances in Polymer Science, 245, 137-172 (2012).

[16] Chiellini E., Corti A., D'Antone S., Solaro R. Biodegradation of poly (vinyl alcohol) based materials. Progress in Polymer Science, 28, No. 6, 963-1014 (2003).

[17] Rudenchyk T. V., Rozhnova R. A., Galatenko N. A., Nechaeva L. Yu. The effects of the model biological medium on the structure and properties of composite materials with levamisole and the dynamics of the release of a drug substance. Voprosy Khimii i Khimicheskoi Tekhnologii, No. 5 , 140-148 (2018).

[18] Rudenchyk T., Rozhnova R., Galatenko N., Narazhayko L., Rudenko A. Study of biodegradation, biocompatibility and bactericidal activity of film materials with tiamulin fumarate based on polyurethaneurea. Chemistry \& Chemical Technology, in press.

[19] Gottlieb D., Shaw P. D. Antibiotics: Volume I Mechanism of action. Springer-Verlad Berlin Heidelberg New York (1967).

[20] Grayson M. L., Crowe S. M., McCarthy J. S., Mills J., Mouton J. W., Norrby S. R., Paterson D. L., Pfaller M. A. Kucers' the use of antibiotics. Sixth Edition: A Clinical Review of Antibacterial, Antifungal, Antiparasitic and Antiviral Drugs, Vol. 1. (2010).

[21] Zhang Y., Yew W-W. Mechanisms of drug resistance in mycobacterium tuberculosis: update 2015. The International Journal of Tuberculosis and Lung Disease, 19, 1276-1289 (2015).

[22] Kulyesh D. V., Rudenchyk T. V., Roznova R. A., Narozhayko L. F., Pinchuk V. D., Kebuladze I. M., Popova N. M. Research and development biocompatibility of composite polymeric materials with cycloserine for medicine. Plastic, Reconstructive and Aesthetic Surgery, No. 1-2, 42-52 (2017).

[23] Cycloserine. Tuberculosis, 88, 100-101 (2008).

[24] Sakka N. El, Gould I. M. Role of old antimicrobial agents in the management of urinary tract infection. Expert Review of Clinical Pharmacology, 9, 1047-1056 (2016).

[25] Brunton L., Lazo J., Parker K. Goodman \& gilman's the pharmacological basis of therapeutics. McGraw-Hill, New York (2005).

[26] Somaraju V. Drugs used in tuberculosis and leprosy. In 'Modern pharmacology with clinical applications' (6th edn) (eds.: Craig C. R., Stitzel R. E.) Lippincott Williams \& Wilkins, Philadelphia, 557-566 (2003).

[27] Baum E. Z., Crespo-Carbone S. M., Foleno B. D., Simon L. D., Guillemont J., Macielag M., Bush K. MurF inhibitors with antibacterial activity: Effect on muropeptide levels. Antimicrobial Agents and Chemotherapy, 53, 3240-3247 (2009).

[28] Vemula H., Ayon N. J., Burton A., Gutheil W. G. Antibiotic effects on methicillin resistant staphylococcus aureus cytoplasmic peptidoglycan intermediate levels and evidence for potential metabolite level regulatory loops. Antimicrobial Agents and Chemotherapy, 61, e02253-16 (2017). 